\title{
ReVISÃo de literatura Papel da memantina no tratamento das perturbações da ansiedade: uma revisão baseada na evidência
}

\author{
The role of memantine in anxiety disorders: an evidence-based review \\ Ana Raquel Martins Marques's, Nivaldo Marins²
}

\section{RESUMO}

Objetivo: Avaliar a eficácia da memantina no tratamento das perturbações da ansiedade. Métodos: Foi realizada uma revisão sistemática na Medline, outras bases de dados baseadas na evidência e Índex das Revistas Médicas Portuguesas, nos últimos 10 anos, em inglês, espanhol e português, utilizando os seguintes termos "MESH memantine", "treatment" e "anxiety disorders". Para avaliar a qualidade dos estudos e a força de recomendação, foi utilizada a escala de Strength of Recommendation Taxonomy da American Family Physician. Resultados: Obtiveram-se 131 artigos, dos quais seis cumpriam os critérios de inclusão: dois ensaios clínicos aleatorizados e controlados (ECAC), uma revisão sistemática (RS), uma norma de orientação clínica (NOC) e dois ensaios clínicos não controlados (ECNC). A NOC não recomenda o uso de memantina no tratamento da perturbação generalizada da ansiedade (PGA) (SOR C), mas recomenda o uso da memantina como tratamento adjuvante de segunda linha na perturbação obsessivo-compulsiva (POC) e como terceira linha na perturbação do estresse pós-traumático (SORT C). Os dois ECAC sugerem que o tratamento adjuvante com memantina melhorou a sintomatologia dos pacientes com POC severa. A RS revelou que o uso da memantina tem alguns benefícios na diminuição dos sintomas da POC. Os dois ECNC sugerem que a memantina pode ser eficaz como tratamento adjuvante em pacientes sintomáticos

\section{Palavras-chave}

Memantina, tratamento, perturbações de ansiedade. apesar de tratamento adequado com antidepressivos convencionais. Conclusão: A evidência atual sugere que o uso de memantina como adjuvante no tratamento da POC parece ser eficaz (SORT B). O uso de memantina no tratamento da PGA não é recomendado (SORT C).

\section{ABSTRACT}

Objective: Review the evidence on the efficacy of memantine in the treatment of the anxiety disorders. Methods: A systematic review of articles published in the last ten years, in Portuguese, English and Spanish, was performed in Medline, other places of Evidence Medicine and Index of Portuguese Databases, using the following MESH terms: memantine, treatment and anxiety disorders. We used the Strength of Recommendation Taxonomy (SORT) scale of American Family Physician to assign levels of evidence and strength of recommendations. Results: A total of 131 papers were identified, and after the application of inclusion criteria, 6 papers remained, including two randomized controlled trials (RCT), one clinical guideline (CG), two open clinical trials and one systematic review (SR). The CG does not recommend the use of memantine in the treatment of the generalized anxiety disorder (SORT C) but

1 UCSP São Mamede - Unidade Local de Saúde de Matosinhos, Portugal.

2 Centro Hospitalar de Conde Ferreira, Porto, Portugal.

Recebido em

$22 / 11 / 2015$

Aprovado em

$28 / 3 / 2016$

Endereço para correspondência: Ana Raquel Martins Marques

Unidade Local de Saúde de Matosinhos Porto, Portugal

E-mail:dr.ana.marques@gmail.com 


\section{Keywords}

Memantine, treatment, anxiety disorders. does recommend the use of memantine as second-line adjunctive therapy for obsessive compulsive disorder and as third-line therapy for post-traumatic stress (SORT C). The two RCT suggest that an add-on of memantine does improve the symptoms of patients suffering from severe obsessive compulsive disorder. The SR showed that the use of memantine had some benefit in relieving obsessive compulsive disorder symptoms. The two other studies showed memantine may be an effective augmentation therapy in patients with anxiety who remain symptomatic despite adequate treatment with conventional antidepressant anxiolytics. Conclusion: The current available evidence suggests the benefit of memantine as an adjunctive therapy for the treatment for obsessive compulsive disorder (SORT B). Most studies does not show the benefit of the use of memantine for the treatment of generalized anxiety disorder (SORT C).

\section{INTRODUÇÃO}

As perturbações da ansiedade são as perturbações psiquiátricas mais frequentes, com prevalência de aproximadamente $31 \%$. O $3^{\circ}$ Censo Psiquiátrico, realizado em Portugal, em 2001, referiu que $12,4 \%$ das pessoas consultadas em instituições psiquiátricas apresentavam transtornos neuróticos, nos quais se incluíam os transtornos da ansiedade'.

As perturbações da ansiedade têm impacto social e funcional elevado, principalmente quando se trata de pacientes com sintomas severos e/ou persistentes, com implicações importantes na qualidade de vida ${ }^{2,3}$. A ansiedade também tem impacto econômico considerável na sociedade, quer pelo absentismo laboral e diminuição da produtividade, quer pelo consumo de recursos de saúde ${ }^{1,2}$.

As perturbações da ansiedade, segundo a classificação DSM-IV (Diagnostic and Statistical Manual of Mental Disorders), são classificadas em várias categorias de diagnóstico: perturbação generalizada da ansiedade (PGA), perturbação obsessivo-compulsiva (POC), fobias, incluindo fobias simples e a fobia social, transtornos de estresse, como a perturbação do estresse pós-traumático, perturbação do pânico, perturbações da ansiedade secundárias a condições físicas ou a iatrogenia, e outras ansiedades.

A necessidade de tratamento é determinada pela severidade e persistência dos sintomas, presença de comorbilidades mentais ou físicas, nível de impacto no funcionamento social do doente, medicação concomitante e antecedentes de resposta e tolerabilidade a tratamentos prévios ${ }^{3}$. Essas perturbações estão associadas a risco aumentado de desenvolver síndrome depressiva maior se não tratadas de forma precoce e adequada².

A resposta terapêutica que se pretende é a melhoria dos sintomas de ansiedade. Os objetivos principais são a remissão da sintomatologia e o regresso ao nível de funcionamento pré-mórbido. Para isso, existem escalas que auxiliam na avaliação da evolução do doente. A escala Clinical Global Impression (CGI) é breve, concisa, de fácil compreensão e pode ser facilmente aplicada em qualquer momento. A Hamilton Anxiety Rating Scale (HARS) permite avaliar os sin- tomas de ansiedade no geral e é habitualmente usada em ensaios clínicos².

As opções de tratamento incluem o tratamento psicológico e o tratamento farmacológico. Todos os pacientes devem receber informação educacional sobre o seu distúrbio, a eficácia e a tolerabilidade das várias opções terapêuticas, os fatores de agravamentos e os sintomas de recaída² ${ }^{2}$ A psicoterapia nem sempre é facilmente acessível e pode requerer tratamentos de duração prolongada 5 .

Vários antidepressivos, incluindo os inibidores seletivos da recaptação da serotonina (SSRIs), inibidores da recaptação da serotonina e norepinefrina (SNRIs), antidepressivos específicos serotoninérgicos e noradrenérgicos (NaSSAs), antidepressivos tricíclicos, inibidores da monoamina oxidase (IMAO) e inibidores reversíveis da MAO A (RIMAs), mostraram ser eficazes no tratamento dos distúrbios da ansiedade ${ }^{2-5}$. Os SSRIs e SNRIs são habitualmente preferidos como tratamento de primeira linha, uma vez que têm menos efeitos adversos e são mais bem tolerados que os antidepressivos tricíclicos e os IMAO. Vários anticonvulsivantes e antipsicóticos atípicos mostraram ser eficazes, mas, por inúmeras razões, incluindo os efeitos adversos associados, são apenas recomendados como tratamento de segunda linha, terceira linha ou adjuvantes à terapêutica².

Entre os fármacos disponíveis no mercado para a ansiedade, já anteriormente referidos, existem ainda alguns que são eficazes em tratamentos de curta duração, tais como as benzodiazepinas, mas cujo abuso pode causar dependência, deterioração cognitiva e risco aumentado de acidentes e quedas ${ }^{2}$.

Verifica-se que menos de $50 \%$ dos pacientes com PGA atingem a remissão com as terapêuticas atualmente disponíveis e aprovadas ${ }^{6}$. Sendo assim, quer pelos efeitos secundários dos fármacos disponíveis, quer pela acessibilidade da psicoterapia, a procura por fármacos alternativos que possam dar resposta a pacientes com distúrbios da ansiedade com sintomas persistentes apresenta-se como uma necessidade.

Achados recentes sugerem que fármacos que têm como alvo a memória e o reforço da cognição podem representar 
uma abordagem alternativa na redução dos sintomas de ansiedade ${ }^{6,7}$.

A memantina é um antagonista não competitivo dos receptores NMDA e é globalmente usada no tratamento da demência de Alzheimer moderada a severa. Os ensaios clínicos com memantina revelaram um perfil de segurança e tolerabilidade favorável. Uma revisão recente indica que os seus efeitos secundários são habitualmente ligeiros a moderados, em comparação com o placebo, e mesmo inexistentes, e incluem tontura, obstipação, cefaleia, hipertensão arterial e sonolência ${ }^{8}$. É predominantemente excretada por via renal. Os pacientes com insuficiência renal (taxa de filtração glomerular de $5-29 \mathrm{ml} / \mathrm{min}$ ) devem limitar a dose a $5 \mathrm{mg}$, duas vezes por dia, de forma a evitar toxicidade por acumulação.

O receptor NMDA é normalmente ativado pela ligação do glutamato, o maior neurotransmissor excitatório do sistema nervoso central. Acredita-se que o aumento do fluxo de iões de cálcio resultante dessa ativação leva à lesão excitotóxica neuronal cerebral. Pensa-se que a ligação da memantina a esse receptor confere neuroproteção, por limitar o dano pela excitotoxicidade glutamatérgica ${ }^{6}$. Em alguns distúrbios da ansiedade como a PGA e a POC, pensa-se que o excesso de glutamato ou uma disfunção glutamatérgica está na base da fisiopatologia dessas perturbações ${ }^{6,7,9}$.

Portanto, o objetivo desta revisão é determinar a evidência científica existente sobre a eficácia da memantina no tratamento das perturbações da ansiedade.

\section{MÉTODOS}

Em julho de 2015, foi realizada uma pesquisa de artigos de revisão, metanálises e ensaios clínicos aleatorizados na Medline e sítios Médicos Baseados na Evidência (The Cochrane Library DARE, Bandolier, Guidelines Finder da National Electronic Library for Health no NHS britânico, National Guideline Clearinghouse, Canadian Medical Association Practice Guidelines InfoBase) e Índex das Revistas Médicas Portuguesas, publicados entre $1^{\circ}$ de julho de 2005 e $1^{\circ}$ de julho de 2015, em inglês, espanhol e português, utilizando-se os seguintes termos "MESH memantine", "treatment" e "anxiety disorders". Foram também usados os termos em português "memantina", "perturbações de ansiedade" e "tratamento".

Foram incluídas todas as publicações cujo estudo incidisse no tratamento das perturbações da ansiedade em adultos com idade igual ou superior a 18 anos. A intervenção avaliada consistiu no uso de memantina em comparação com o placebo (em associação ou não com outras classes terapêuticas recomendadas). Como desfechos/resultados de eficácia, definiu-se o alívio da sintomatologia; como desfecho/ resultado de segurança, definiu-se a incidência de efeitos adversos. Foi excluída a população em idade pediátrica, em razão da incidência baixa dessa patologia nesse grupo etário; também foram excluídos artigos de opinião, editoriais, letters e notícias, artigos que não se encontraram na forma integral, artigos repetidos e aqueles que não cumpriam os critérios de inclusão previamente descritos.

Para avaliar a qualidade dos estudos e a força de recomendação, foi utilizada a escala de Strength of Recommendation Taxonomy da American Family Physician. Trata-se de uma escala que tem em conta a qualidade, a quantidade e a consistência da evidência presente nos estudos. A taxonomia enfatiza a evidência orientada para o doente que mede a morbilidade e a mortalidade. Uma força de recomendação nível A é baseada em múltiplos ensaios clínicos aleatorizados consistentes e diretamente relevantes, a de nível B é baseada em alguma evidência de ensaios clínicos aleatorizados, mas cientificamente não ótimos, e a de nível C é baseada em consensos/opiniões de peritos/evidência orientada para a doença, e não para o doente.

\section{RESULTADOS}

Da pesquisa bibliográfica realizada, resultou um total de 131 artigos, tendo sido apenas incluídos seis artigos: dois ensaios clínicos aleatorizados controlados, dois ensaios clínicos não controlados, uma revisão sistemática e uma norma de orientação clínica. O fluxograma de seleção dos estudos está representado na figura 1.

\section{Seção Resultados}

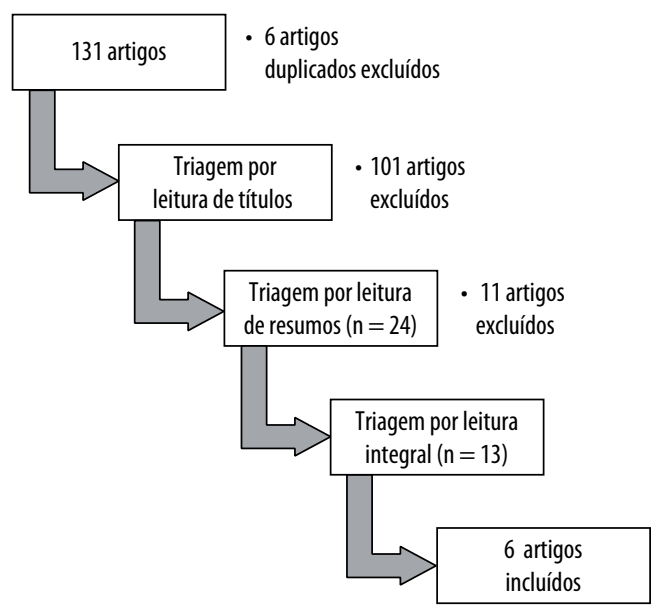

Figura 1. Fluxograma de seleção dos estudos.

A maioria dos artigos foi excluída após leitura do título, por estes não estarem relacionados diretamente com o tema. A qualidade dos artigos presentes na revisão sistemática foi avaliada para a determinação do nível de evidência da revisão, não tendo sido considerados individualmente para a atribuição da força de recomendação final. Essa avaliação resumida pode ser consultada no quadro 1. Quanto a normas de orientação clínica, a pesquisa revelou apenas um resultado (Quadro 2). 
Quadro 1. Resumo das conclusões dos artigos incluídos e nível de evidência segundo a escala SORT

\begin{tabular}{|c|c|c|c|c|c|}
\hline Autor (ano) & Tipo de estudo & População/intervenção & Desfechos/resultados & Conclusão & NE \\
\hline Haghighi et al. $(2013)^{10}$ & ECAC & $\begin{array}{l}\mathrm{N}=40 \text { com POC (14-40 } \\
\text { anos) } \\
\text { Os dois grupos medicados } \\
\text { com SSRI ou clomipramina } \\
\text { Intervenção: Grupo } 1 \text { com } \\
\text { memantina vs. Grupo } 2 \text { com } \\
\text { placebo } \\
\text { Follow-up: } 12 \text { semanas }\end{array}$ & $\begin{array}{l}\text { Redução dos sintomas de } \\
\text { POC em } \geq 35 \% \text { com base na } \\
\text { Y-BOCS. }\end{array}$ & $\begin{array}{l}0 \text { tratamento adjuvante com memantina } \\
\text { alcançou diferenças estatisticamente } \\
\text { significativas entre os grupos na redução da } \\
\text { sintomatologia, com melhoria } \geq 35 \% \text { em } 31 \% \\
\text { dos pacientes, com base na Y-B0CS, quando } \\
\text { comparado com o grupo placebo. }\end{array}$ & 2 \\
\hline Ghaleiha et al. (2013)11 & ECAC & $\begin{array}{l}\mathrm{N}=42 \text { com POC moderada a } \\
\text { severa ( } 18-60 \text { anos) } \\
\text { Intervenção: Memantina } \\
10 \mathrm{mg} / \text { dia na } 1^{\text {a } ~ s e m a n a ~ e ~} \\
20 \mathrm{mg} / \text { dia nas restantes } \\
\text { semanas + fluvoxamina vs. } \\
\text { placebo + fluvoxamina } \\
\text { Follow-up: } 8 \text { semanas }\end{array}$ & $\begin{array}{l}\text { Avaliar a eficácia e } \\
\text { a tolerabilidade da } \\
\text { memantina como adjuvante } \\
\text { do tratamento de pacientes } \\
\text { com POC moderada a } \\
\text { severa. } \\
\text { Redução dos sintomas em } \\
\geq 35 \% \text { com base na escala } \\
\text { Y-BOCS. }\end{array}$ & $\begin{array}{l}\text { Todos os pacientes do grupo memantina } \\
\text { tiveram redução dos sintomas em } \geq 35 \% \\
\text { ( } p<0,001 \text { ). No final do ensaio, } 89 \% \text { dos } \\
\text { pacientes do grupo da memantina, comparados } \\
\text { com } 6 \% \text { do grupo placebo, obtiveram remissão } \\
\text { completa dos sintomas. } \\
\text { A frequência dos efeitos adversos não mostrou } \\
\text { diferenças estatisticamente significativas nos } \\
\text { dois grupos. }\end{array}$ & 1 \\
\hline Schwartz et al. $(2012)^{7}$ & $\begin{array}{l}\text { Ensaio clínico não } \\
\text { controlado }\end{array}$ & $\begin{array}{l}\mathrm{N}=15 \\
\text { com PAG } \\
\text { Intervenção: Memantina de } \\
5 \text { a } 20 \mathrm{mg} / \text { dia } \\
\text { Follow-up: } 10 \text { semanas }\end{array}$ & $\begin{array}{l}\text { Avaliar o possível papel da } \\
\text { memantina no tratamento } \\
\text { das perturbações de } \\
\text { ansiedade. } \\
\text { Redução da sintomatologia } \\
\text { em } \geq 50 \% \text { avaliada pela } \\
\text { HARS. }\end{array}$ & $\begin{array}{l}\text { Diminuição em } \geq 50 \% \text { dos sintomas de } \\
\text { ansiedade em } 60 \% \text { dos pacientes. Cerca de } 40 \% \\
\text { alcançaram a remissão (HARS }<7 \text { ). }\end{array}$ & 3 \\
\hline Feusner et al. (2009) ${ }^{12}$ & $\begin{array}{l}\text { Ensaio clínico não } \\
\text { controlado }\end{array}$ & $\begin{array}{l}\mathrm{N}=17 \text { (10 com POC e } 7 \text { com } \\
\text { PAG) (18 e } 64 \text { anos) } \\
\text { Intervenção: memantina no } \\
\text { máximo de } 10 \mathrm{mg} 2 \text { } 2 \text { /dia em } \\
\text { monoterapia ou associada a } \\
\text { terapêutica ja existente } \\
\text { Follow-up: } 12 \text { semanas }\end{array}$ & $\begin{array}{l}\text { Comparar a eficácia e a } \\
\text { segurança da memantina na } \\
\text { POC e na PAG. } \\
\text { Desfecho primário: } \\
\text { diminuição da } \\
\text { sintomatologia em } \geq 35 \% \\
\text { pela Y-BOCS, no grupo } \\
\text { com POC, e em } \geq 50 \% \text { pela } \\
\text { HARS, no grupo com PAG, } \\
\text { e em ambos os grupos, na } \\
\text { classificação de "muito } \\
\text { melhorado" ou "melhorado" } \\
\text { na escala CGI-I. Desfecho } \\
\text { secundário: monitorização } \\
\text { da segurança. }\end{array}$ & $\begin{array}{l}\text { A memantina parece ser eficaz no grupo com } \\
\text { POC. } \\
\text { No grupo com PAG nenhum participante foi } \\
\text { classificado como responsivo ao tratamento, } \\
\text { pois nenhum teve redução em } \geq 50 \% \text { dos } \\
\text { sintomas pela HARS. }\end{array}$ & 3 \\
\hline \multirow[t]{3}{*}{ Zdanys e Tampi (2008) $)^{13}$} & \multicolumn{2}{|c|}{ Revisão sistemática (2 ensaios clínicos não controlados) } & & & 2 \\
\hline & $\begin{array}{l}\text { Poyurovsky et al., } 2005 \\
\text { (relato de caso) }\end{array}$ & $\begin{array}{l}\mathrm{N}=1 \text { com } \mathrm{POC} \\
\text { Intervenção: } \\
\text { memantina } 20 \mathrm{mg} / \mathrm{dia}+ \\
\text { clomipramina } 300 \mathrm{mg} / \mathrm{dia}+ \\
\text { sulpiride } 400 \mathrm{mg} / \mathrm{dia} \\
\text { Follow-up: } 2 \text { semanas }\end{array}$ & $\begin{array}{l}\text { Desfecho: melhoria da } \\
\text { sintomatologia no doente } \\
\text { com POC medida pela } \\
\text { Y-BOCS. }\end{array}$ & $\begin{array}{l}\text { Diminuição na severidade dos sintomas } \\
\text { no doente com POC. Sem efeitos adversos } \\
\text { relatados. }\end{array}$ & \\
\hline & $\begin{array}{l}\text { Pasquini e Biondi, } 2006 \\
\text { (relato de caso) }\end{array}$ & $\begin{array}{l}\mathrm{N}=2 \text { com POC. } \\
\text { Intervenção: } \\
\text { memantina } 15 \mathrm{mg} / \mathrm{dia}+ \\
\text { venlafaxina } 300 \mathrm{mg} / \mathrm{dia} \text { ou } \\
\text { citalopram } 60 \mathrm{mg} / \text { dia } \\
\text { Follow-up: } 3 \text { semanas }\end{array}$ & $\begin{array}{l}\text { Desfecho: melhoria da } \\
\text { sintomatologia no doente } \\
\text { com POC medida pela } \\
\text { Y-BOCS. }\end{array}$ & $\begin{array}{l}\text { Doente 1: sem melhoria; } \\
\text { Doente 2: diminuição da sintomatologia de POC. } \\
\text { Estado disfórico relatado no doente } 2 \text { com dose } \\
\text { de memantina de } 15 \mathrm{mg} / \text { dia. }\end{array}$ & \\
\hline
\end{tabular}

ECAC: ensaio clínico aleatorizado controlado; N: número de participantes; POC: perturbação obsessivo-compulsiva; PAG: perturbação da ansiedade generalizada; Y-BOCS: Yale-Brown Obsessive Compulsive Scale; HARS: Hamilton Anxiety Rating Scale; (GI-I: Clinical Global Impression - Improvement and Severity Scales.

Quadro 2. Norma de Orientação Clínica

\begin{tabular}{lll}
\hline NOC & Ano & Conclusão \\
\hline Katzman et al. ${ }^{2}$ & 2014 & Não recomenda o uso da memantina no tratamento da PAG por falta \\
& & $\begin{array}{l}\text { de evidência de sua eficácia - (SORT C). } \\
\text { Recomenda o uso de memantina como adjuvante de segunda linha } \\
\text { no tratamento da POC e como tratamento de terceira linha na } \\
\text { perturbação do estresse pós-traumático - (SORT C) }\end{array}$ \\
\hline
\end{tabular}

NOC: Norma de Orientação Clínica; NE: nível de evidência; SORT: força de recomendação; POC: perturbação obsessivo-compulsiva; PAG: perturbação da ansiedade generalizada. 
O ECAC de Haghighi et al. ${ }^{10}$, publicado em 2013, foi desenhado com o objetivo de avaliar a eficácia da memantina como adjuvante no tratamento da POC, comparativamente ao placebo, na melhoria da sintomatologia. Foi constituída uma amostra de 40 pacientes, com idades compreendidas entre 18 e 40 anos, com diagnóstico de POC, segundo a classificação DSM-IV-TR (American Psychiatric Association 2000) e com pontuação $\geq 21$ pontos na Yale - Brown Obsessive Compulsive Scale (Y-BOCS). Essa amostra foi aleatorizada em dois grupos: ao grupo 1 foi administrada memantina (5-10 mg/dia); ao grupo 2 foi administrado placebo (5-10 $\mathrm{mg} / \mathrm{dia}$ ). Os dois grupos, na semana anterior e durante o estudo, receberam tratamento com um SSRI ou clomipramina. Ao longo das 12 semanas de seguimento, houve desistência de 11 pacientes, tendo concluído o estudo 72,5\% da amostra. A sintomatologia de POC foi avaliada por meio da Y-BOCS em quatro períodos de tempo - no início do estudo e após 4, 8 e 12 semanas. Os autores definiram como desfecho primário uma redução dos sintomas em $\geq 35 \%$ como resposta completa ao tratamento, uma redução de 25\%-35\% como resposta parcial e se $<25 \%$ como sem resposta, com base na Y-BOCS, nas 12 semanas. Eles concluíram que o tratamento adjuvante com memantina alcançou diferenças estatisticamente significativas entre os grupos na melhoria da sintomatologia quando comparado com o placebo. Registraram-se efeitos adversos em apenas dois pacientes, sem gravidade.

O ECAC de Ghaleiha et al. ${ }^{11}$, publicado em 2013, foi desenhado com o objetivo de avaliar a eficácia e a tolerabilidade da memantina como adjuvante no tratamento da POC moderada a severa, comparativamente ao placebo, na melhoria dos sintomas. Uma amostra de 42 pacientes, com idades compreendidas entre 18 e 60 anos, com diagnóstico de POC, segundo a classificação DSM-IV-TR (American Psychiatric Association 2000) e com pontuação $\geq 21$ pontos na Y-BOCS, foi aleatorizada em dois grupos: ao grupo 1 foi administrada memantina $(10 \mathrm{mg} /$ dia na primeira semana e $20 \mathrm{mg} /$ dia nas semanas restantes); ao grupo 2 foi administrado placebo. Ambos os grupos estavam medicados com fluvoxamina. Após oito semanas, manteve-se seguimento de mais de $90 \%$ da amostra. Os autores definiram como desfecho primário uma resposta completa se redução dos sintomas fosse igual ou superior a 35\%, uma resposta parcial se redução fosse de $25 \%-35 \%$ e sem resposta se redução fosse $<25 \%$, com base na pontuação obtida pela Y-BOCS. Passadas oito semanas, todos os pacientes do grupo memantina corresponderam aos critérios de resposta completa ou parcial ao tratamento $(p<0,001)$. Em torno de $89 \%$ dos pacientes do grupo da memantina, comparados com $6 \%$ do grupo placebo, tiveram remissão completa dos sintomas. A frequência dos efeitos adversos não mostrou diferenças estatisticamente significativas nos dois grupos.
Schwartz et al. ${ }^{9}$ publicaram um estudo em 2012, que foi desenhado com o objetivo de avaliar o possível papel da memantina no tratamento das perturbações de ansiedade. Uma amostra de 15 pacientes, com média de idade de 44 anos, com diagnóstico de PAG, iniciou o tratamento com memantina entre 5 e $20 \mathrm{mg} / \mathrm{dia}$, durante 10 semanas. A sintomatologia foi avaliada por meio da HARS. Os autores definiram como desfecho primário uma redução da sintomatologia, avaliada pela HARS, superior ou igual a 50\%, e a remissão completa foi considerada se a pontuação na HARS fosse inferior a 7. Passadas 10 semanas, verificou-se diminuição dos sintomas de ansiedade em $60 \%$ dos pacientes, e cerca de $40 \%$ entraram em remissão.

O ensaio clínico não controlado de Feusner et al. ${ }^{12}$, publicado em 2009, foi desenhado com o objetivo de comparar a eficácia e a segurança da memantina na POC e na PAG. Uma amostra de 17 pacientes, com idade compreendida entre 18 e 64 anos, com diagnóstico de POC $(n=10)$ e PAG $(n=7)$ iniciou tratamento com memantina entre 10 e $30 \mathrm{mg} / \mathrm{dia}$, durante 12 semanas. Doze dos participantes (70\%) já tomavam outra medicação psicotrópica previamente ao estudo (SSRI n $=10$ e benzodiazepina $n=1$, e ambos $n=1$ ) e a mantiveram no decorrer das 12 semanas. O desfecho primário foi diminuir a sintomatologia obsessivo-compulsiva na POC, medida pela redução em $\geq 35$ pontos na Y-BOCS, e reduzir em $\geq 50 \%$ os sintomas de ansiedade, segundo a HARS, no grupo com PAG. Em ambos os grupos, pretendeu-se ainda melhorar a qualidade de vida global medida por meio da escala CGI-I (Clinical Global Impression - Improvement and Severity Scales), com classificação de "muito melhorado" ou "melhorado". Como desfecho secundário, monitorizou-se a segurança do fármaco. Passadas as 12 semanas, o grupo com POC mostrou melhoria significativa, com redução de $40,6 \%$ na pontuação na Y-BOCS ( $p<0,001)$. No grupo com PAG, nenhum participante foi classificado como responsivo ao tratamento, pois nenhum teve redução $\geq 50 \%$ dos sintomas pela HARS.

Zdanys e Tampi ${ }^{13}$ realizaram uma revisão sistemática, em 2008, sobre o uso off-label da memantina em distúrbios psiquiátricos como depressão, esquizofrenia, POC, doença bipolar, entre outros. Relativamente à seção sobre a POC, foi incluído na revisão sistemática um total de dois estudos referentes à utilização da memantina no tratamento da POC: dois estudos de relato de caso. Nos dois estudos, três pacientes receberam tratamento adjuvante com a memantina; desses, apenas dois demonstraram melhoria sintomática.

A NOC canadense², para a abordagem da PAG, do estresse pós-traumático e da POC, publicadas em 2014, não recomenda o uso da memantina no tratamento da PAG, por falta de evidência da sua eficácia. No entanto, recomenda o uso de memantina como adjuvante de segunda linha no tratamento da POC e como tratamento de terceira linha na perturbação do estresse pós-traumático. 


\section{DISCUSSÃO}

Ao ECAC de Haghighi et al. ${ }^{10}$, publicado em 2013, foi atribuído um nível de evidência 2 (NE 2). Esse estudo concluiu que o tratamento adjuvante com memantina alcançou diferenças estatisticamente significativas entre os grupos na melhoria da sintomatologia quando comparado com o placebo. Apesar dos resultados, várias limitações que não permitem a generalização dos achados à população geral devem ser consideradas, tais como o tamanho amostral reduzido com follow-up de menos de $80 \%$ dos pacientes e o fato de os sintomas depressivos e de ansiedade não terem sido contabilizados e poderem tratar-se de fatores de confundimento na aplicação da Y-BOCS.

Ao ECAC de Ghaleiha et al. ${ }^{11}$, publicado em 2013, foi atribuído um nível de evidência 1 (NE 1). Os autores concluíram que a memantina levou a melhorias significativas, na maioria dos casos com resposta completa ou parcial ao tratamento, nos pacientes com POC moderada a severa. Trata-se de um estudo de elevada qualidade, duplamente cego, randomizado, com tamanho amostral adequado, com intenção de tratar e com seguimento de mais de $90 \%$ da amostra. No entanto, o tempo de seguimento dos pacientes foi reduzido, não permitindo aferir efeitos a longo prazo.

Ao estudo de Schwartz et al. ${ }^{9}$, publicado em 2011, foi atribuído um nível de evidência 3 (NE 3). Esse estudo apresenta várias limitações, pois trata-se de um ensaio clínico não aleatorizado, com uma amostra de utentes de pequenas dimensões, com tempo de follow-up reduzido e com seguimento de apenas 53\% da amostra.

Foi atribuído um nível de evidência 3 (NE 3) ao ensaio clínico não controlado de Feusner et al. ${ }^{12}$, publicado em 2009. O grupo com POC mostrou melhoria significativa e o grupo com PAG não se mostrou responsivo ao tratamento com memantina. Apesar dos resultados positivos no grupo com POC, há várias limitações a ter em conta nesse estudo. O fato de alguns pacientes estarem sob outros tratamentos e haver variedade de dosagens deles pode ser um fator de confundimento nos resultados no grupo com POC. O tamanho da amostra do grupo com PAG é reduzido, o que pode justificar a aparente falta de eficácia da memantina nesses pacientes.

O estudo de Zdanys e Tampi ${ }^{13}$ é uma revisão sistemática, publicado em 2008, ao qual foi atribuído um nível de evidência 2 (NE 2). Relativamente à seção sobre a POC, eles incluíram dois estudos de relato de caso com três pacientes, dos quais apenas dois demonstraram melhoria sintomática. Apesar de se tratar de uma revisão sistemática, ele mostrou ter várias limitações, nomeadamente por incluir apenas dois estudos que metodologicamente não são de boa qualidade, com tamanhos amostrais muito reduzidos e achados inconsistentes.

Da pesquisa, resultou ainda uma revisão sistemática de Barton et al. ${ }^{14}$, de 2014, com o objetivo de avaliar a eficácia do tratamento farmacológico e psicológico e terapias alternativas em idosos com perturbações de ansiedade. Os autores excluíram esse estudo, pois não foram encontrados estudos clínicos randomizados e controlados ou estudos prospectivos observacionais comparativos nessa faixa etária da população.

Às conclusões da NOC canadense ${ }^{2}$ para a abordagem da PAG, do estresse pós-traumático e da POC, publicadas em 2014, foi atribuída uma força de recomendação C, por se basearem em estudos heterogêneos e de pouca qualidade e robustez.

Assim, a revisão realizada possui pontos fortes a serem referidos. A revisão da literatura foi realizada com estudos dos últimos dez anos, abrangendo, dessa forma, toda a literatura, mesmo a mais antiga, com dados importantes. Outro ponto a ser considerado foi a inclusão de ensaios clínicos controlados e randomizados e a existência de uma revisão sistemática sobre o tema, apesar de ela ser baseada em estudos de fraca qualidade. Foram incluídos ensaios clínicos não controlados, mas, apesar de serem menos robustos, por causa da escassez de ensaios clínicos nessa temática, os autores optaram por não excluir a informação deles.

O fato de os estudos serem globalmente heterogêneos, nomeadamente no que se refere ao desenho e à qualidade deles, à dose da memantina, à diversidade de fármacos com os quais foi associada nos diversos estudos, constituiu das principais limitações desta revisão.

\section{CONCLUSÃO}

Muito embora tenham sido pesquisadas múltiplas bases de dados, os estudos sobre esse tópico são escassos e a sua evidência limitada. Em primeiro lugar, existem poucos estudos sobre o tratamento das perturbações da ansiedade com a memantina. Apesar da publicação de uma NOC canadense recente (2014), esta é baseada em estudos globalmente heterogêneos, nomeadamente no que se refere ao desenho e à qualidade deles, à dose de memantina usada e à diversidade de fármacos com os quais foi associada nos diversos estudos, colocando várias limitações. São necessários mais estudos para clarificar a consistência de alguns resultados. Também o fato de ser um fármaco dispendioso no Serviço Nacional de Saúde português pode atuar como limitação na prescrição, pelas condições econômicas que o país atravessa.

Perante os resultados obtidos:

- Parece razoável recomendar o uso de memantina como adjuvante no tratamento da POC. Essa conclusão é apoiada por uma norma de orientação clínica de consenso de expertos, dois ensaios clínicos randomizados e controlados (com NE 1 e 2) e uma revisão sistemática (com NE 2), pelo que Ihe atribuímos uma força de recomendação $\mathbf{B}$. 
- O uso de memantina no tratamento da PAG não é recomendado e esta conclusão é apoiada por uma norma de orientação clínica e dois ensaios clínicos não controlados (com NE 3), pelo que lhe atribuímos uma força de recomendação $\mathbf{C}$.

No que respeita à segurança, a memantina mostrou-se tão segura quanto o placebo relativamente à ocorrência de efeitos adversos.

Futuramente será imprescindível a realização de mais estudos, com amostras de grandes dimensões, homogêneos e de boa qualidade, que avaliem o custo/benefício da memantina como tratamento ou adjuvante, ou mesmo como tratamento em monoterapia, no alívio da sintomatologia nos distúrbios da ansiedade, com o intuito de encorajar ou desencorajar o seu uso.

\section{CONTRIBUIÇÕES INDIVIDUAIS}

Ana Raquel Marques - Contribuiu na concepção do estudo, colheita dos dados, redação do manuscrito, análise e interpretação dos dados, revisão e aprovação da versão final do manuscrito.

Nivaldo Marins - Contribuiu na concepção do estudo, revisão e aprovação final do manuscrito.

\section{CONFLITOS DE INTERESSE}

Não há conflitos de interesse a serem declarados.

\section{REFERÊNCIAS}

1. Bento A, Carreira M, Heitor MJ. $3^{\circ}$ Censo Psiquiátrico de 2001. Síntese dos resultados preliminares. Lisboa: Ministério da Saúde/Direção Geral de Saúde. Disponível em: <http:// www.dgs.pt>. Acesso em: 21 out. 2015.
2. Katzman MA, Bleau P, Blier P, Chokka P, Kjernisted K, Van Ameringen M; Canadian Anxiety Guidelines Initiative Group on behalf of the Anxiety Disorders Association of Canada/Association Canadienne des troubles anxieux and McGill University, et al. Canadian clinical practice guidelines for the management of anxiety, posttraumatic stress and obsessivecompulsive disorders. BMC Psychiatry. 2014;14 Suppl 1:S1.

3. Baldwin DS, Anderson IM, Nutt DJ, Bandelow B, Bond A, Davidson JR, et al.; British Association for Psychopharmacology. Evidence-based guidelines for the pharmacological treatment of anxiety disorders: recommendations from the British Association for Psychopharmacology. J Psychopharmacol. 2005;19(6):567-96.

4. Bandelow B, Sher L, Bunevicius R, Hollander E, Kasper S, Zohar J, et al.; WFSBP Task Force on Mental Disorders in Primary Care; WFSBP Task Force on Anxiety Disorders, OCD and PTSD. Guidelines for the pharmacological treatment of anxiety disorders, obsessive-compulsive disorder and posttraumatic stress disorder in primary care. Int J Psychiatry Clin Pract. 2012;16(2):77-84.

5. Nunes A, Sousa M. Utilização da valeriana nas perturbações de ansiedade e do sono: qual a melhor evidência? Acta Med Port. 2011;24:961-6.

6. Nandhra HS, Murphy CL, Sule A. Novel pharmacological agents targeting memory and cognition in the treatment of anxiety disorders. Hum Psychopharmacol. 2013;28(6):538-43.

7. National Collaborating Centre for Mental Health. The Royal College of Psychiatrists. Generalised anxiety disorder in adults: management in primary, Secondary and Community Care. The British Psychological Society and The Royal College of Psychiatrists National Clinical Guideline Number 113.

8. Sani G, Serra G, Kotzalidis GD, Romano S, Tamorri SM, Manfredi G, et al. The role of memantine in the treatment of psychiatric disorders other than the dementias: a review of current preclinical and clinical evidence. CNS Drugs. 2012;26(8):663-90.

9. Schwartz T, Siddiqui U, Raza S. Memantine as an augmentation therapy for anxiety disorders. Case Rep Psychiatry. 2012;2012;749796:1-3.

10. Haghighi M, Jahangard L, Mohammad-Beigi H, Bajoghli H, Hafezian H, Rahimi A, et al. In a double-blind, randomized and placebo-controlled trial, adjuvant memantine improved symptoms in inpatients suffering from refractory obsessive-compulsive disorders (OCD). Psychopharmacology (Berl). 2013;228(4):633-40

11. Ghaleiha A, Entezari N, Modabbernia A, Najand B, Askari N, Tabrizi M, et al. Memantine add-on in moderate to severe obsessive-compulsive disorder: randomized double-blind placebo-controlled study. J Psychiatr Res. 2013;47(2):175-80.

12. Feusner JD, Kerwin L, Saxena S, Bystritsky A. Differential efficacy of memantine for obsessive-compulsive disorder vs. generalized anxiety disorder: an open-label trial. Psychopharmacol Bull. 2009;42(1):81-93.

13. Zdanys K, Tampi RR. A systematic review of off-label uses of memantine for psychiatric disorders. Prog Neuropsychopharmacol Biol Psychiatry. 2008;32(6):1362-74.

14. Barton S, Karner C, Salih F, Baldwin DS, Edwards SJ. Clinical effectiveness of interventions for treatment-resistant anxiety in older people: a systematic review. Health Technol Assess. 2014;18(50):1-59. 